\title{
Direct Interspecies Electron Transfer Mediated by Graphene Oxide-Based Materials
}

\section{OPEN ACCESS}

Edited by:

Amelia-Elena Rotaru,

University of Southern Denmark,

Denmark

Reviewed by:

Carolina Cruz Viggi,

Water Research Institute (IRSA), Italy

Oona Snoeyenbos-West,

Michigan State University,

United States

${ }^{*}$ Correspondence:

Souichiro Kato

s.katou@aist.go.jp

tPresent address:

Eijiro Miyako,

School of Materials Science, Japan

Advanced Institute of Science

and Technology, Nomi, Japan

Specialty section:

This article was submitted to Microbial Physiology and Metabolism,

a section of the journal

Frontiers in Microbiology

Received: 18 September 2019

Accepted: 19 December 2019

Published: 17 January 2020

Citation:

Igarashi K, Miyako E and Kato S (2020) Direct Interspecies Electron Transfer Mediated by Graphene Oxide-Based Materials.

Front. Microbiol. 10:3068. doi: 10.3389/fmicb.2019.03068

\author{
Kensuke Igarashi', Eijiro Miyako ${ }^{2 \dagger}$ and Souichiro Kato ${ }^{1,3 *}$ \\ ${ }^{1}$ Bioproduction Research Institute, National Institute of Advanced Industrial Science and Technology, Sapporo, Japan, \\ ${ }^{2}$ Nanomaterials Research Institute, AIST, Tsukuba, Japan, ${ }^{3}$ Division of Applied Bioscience, Graduate School of Agriculture, \\ Hokkaido University, Sapporo, Japan
}

Conductive materials are known to promote direct interspecies electron transfer (DIET) by electrically bridging microbial cells. Previous studies have suggested that supplementation of graphene oxide (GO) based materials, including $\mathrm{GO}$, and reduced GO (rGO), to anaerobic microbial communities, can promote DIET. This promotion mechanism is thought to be involved in electron transfer via $\mathrm{rGO}$ or biologically formed rGO. However, concrete evidence that rGO directly promotes DIET is still lacking. Furthermore, the effects of the physicochemical properties of GO-based materials on DIET efficiency have not been elucidated. In the current work, we investigated whether chemically and biologically reduced GO compounds can promote DIET in a defined model coculture system, and also examined the effects of surface properties on DIET-promoting efficiency. Supplementation of GO to a defined DIET coculture composed of an ethanol-oxidizing electron producer Geobacter metallireducens and a methane-producing electron consumer Methanosarcina barkeri promoted methane production from ethanol. X-ray photoelectron spectroscopy revealed that GO was reduced to rGO during cultivation by $G$. metallireducens activity. The stoichiometry of methane production from ethanol and the isotope labeling experiments clearly showed that biologically reduced GO induced DIET-mediated syntrophic methanogenesis. We also assessed the DIET-promoting efficiency of chemically reduced GO and its derivatives, including hydrophilic amine-functionalized $\mathrm{rGO}\left(\mathrm{rGO}-\mathrm{NH}_{2}\right)$ and hydrophobic octadecylamine-functionalized rGO (rGO-ODA). While all tested rGO derivatives induced DIET, the rGO derivatives with higher hydrophilicity showed higher DIET-promoting efficiency. Optical microscope observation revealed that microbial cells, in particular, G. metallireducens, more quickly adhered to more hydrophilic GO-based materials. The superior ability to recruit microbial cells is a critical feature of the higher DIET-promoting efficiency of the hydrophilic materials. This study demonstrates that biologically and chemically reduced GO can promote DIET-mediated syntrophic methanogenesis. Our results also suggested that the surface hydrophilicity (i.e., affinity toward microbial cells) is one of the important determinants of the DIET-promoting efficiencies. These observations will provide useful guidance for the selection of conductive particles for the improvement of methanogenesis in anaerobic digesters.

Keywords: GO-based materials, DIET, microbial communities, Geobacter metallireducens, Methanosarcina barkeri 


\section{INTRODUCTION}

Direct interspecies electron transfer (DIET) plays a crucial role in the anaerobic biodegradation process, in which electrons released from electron-producing microorganisms (e.g., Geobacter species) are transferred directly to electronconsuming microorganisms (e.g., Methanosarcina species) (Reguera et al., 2005; Lovley, 2011; Kato, 2015; Kouzuma et al., 2015). DIET mechanism has been extensively studied using a defined coculture system composed of electroactive microorganisms, such as Geobacter species and members of Methanosarcinales methanogens (Summers et al., 2010; Rotaru et al., 2014a,b). Rotaru et al. (2014a) used DIET coculture of Geobacter metallireducens and Methanosarcina barkeri to report basic characteristics of the model DIET coculture and determined that long-range electron transfer via pilin is necessary for DIET. They also showed that a pilin-deficient $G$. metallireducens mutant failed to establish DIET with $M$. barkeri, but an amendment of electroconductive-activated carbon permitted the mutant to establish DIET. This result suggests that conductive materials can electrically connect cells and promote DIET.

The contribution of DIET in complex microbial communities has been studied in order to evaluate the potential of DIET to increase reaction performance of anaerobic digestor and microbial fuel cells (Liu et al., 2012; Li et al., 2015; Dang et al., 2016; Lee et al., 2016; Lin et al., 2017; Tian et al., 2017). Supplementation of conductive materials to anaerobic methanogenic microbial communities promotes DIET by functioning as an electron conduit connecting electroactive cells. Various conductive materials have been assayed for DIETpromoting efficiency so far. These materials include iron oxides (Kato et al., 2012a,b; Baek et al., 2015; Yamada et al., 2015; Yin et al., 2016), activated carbon (Lee et al., 2016), biochar (Chen et al., 2014b), carbon cloth (Chen et al., 2014a), and graphite (Dang et al., 2016). Recently, electroconductive carbon nanomaterials have been extensively studied as new types of multifunctional conductive materials, owing to their unique physical and chemical characteristics (Kamran et al., 2019; Sang et al., 2019).

Graphene, a carbon nanomaterial composed of a single atomic layer of two-dimensional carbon atoms with a honeycomb structure, has attracted considerable attention because of various physicochemical properties, such as significantly high surface area, superior thermal/electrical conductivity, and photocatalytic activity (Novoselov et al., 2004; Kostarelos and Novoselov, 2014). Graphene oxide (GO) is the precursor for large-scale synthesis of graphene (Hummers and Offeman, 1958; Li et al., 2014). GO surface bears various oxygen-containing functional groups, such as hydroxyl, epoxide, diol, and carbonyl groups (Kostarelos and Novoselov, 2014). Due to these functional groups, the GO surface is hydrophilic and thus exhibits high water dispersibility and good biocompatibility, though it lacks electroconductivity (Li et al., 2014; Zhou and Liang, 2014). Chemical reduction of GO produces reduced GO (rGO), which exhibits similar physicochemical properties to pristine graphene. Thus, a number of studies have employed GO or rGO instead of pristine graphene to analyze the interactions occurring with mammalian and bacterial cells (Hu et al., 2010; Li et al., 2014; Zhou and Liang, 2014), whose potential large-scale applications could be considerable.

It has been reported that GO is biologically reduced by several microorganisms capable of extracellular electron transfer such as Geobacter species (Goto and Yoshida, 2016; Yoshida et al., 2016a,b) and Shewanella oneidensis (Jiao et al., 2011; Wang et al., 2011; Yong et al., 2014), which results in the formation of electroconductive complexes composed of rGO and microorganisms (Yong et al., 2014; Yoshida et al., 2016a). Besides, several studies have reported that supplementation of rGO to anaerobic digester promotes methane production rate and increases the population of electroactive microorganisms (e.g., Geobacter and Methanosarcina species) (Lin et al., 2017; Tian et al., 2017). These studies indicate that rGO enhances the microbial activity of anaerobic microbial communities, most likely via the promotion of DIET. However, direct evidence showing that chemically or biologically formed rGO can promote DIET has yet to be reported. Furthermore, the effects of rGO physicochemical properties on DIET efficiency remains unknown. In the present study, we examined DIET-promoting efficiency of GO using a defined DIET coculture. Besides, the effect of different physicochemical properties on DIETpromoting efficiency was assessed by chemically modified rGO, namely hydrophilic amine-functionalized rGO $\left(\mathrm{rGO}-\mathrm{NH}_{2}\right)$ and hydrophobic octadecylamine-functionalized rGO (rGO-ODA).

\section{MATERIALS AND METHODS}

\section{Microorganisms and Cultivations}

Geobacter metallireducens (DSM7210 ${ }^{\mathrm{T}}$ ) and $M$. barkeri $\left(\mathrm{DSM} 800^{\mathrm{T}}\right)$ were obtained from the Deutsche Sammlung von Mikroorganismen und Zellkulturen GmbH (Braunschweig, Germany). All anaerobic cultivations in this study were carried out using a sealed glass bottle (68 $\mathrm{mL}$ in capacity), as described previously (Kato et al., 2015), filled with $10 \mathrm{~mL}$ of IET-P medium under an atmosphere of $100 \mathrm{kPa}$ of $\mathrm{N}_{2}+\mathrm{CO}_{2}(80: 20)$ at $30^{\circ} \mathrm{C}$ in the dark without agitation. The medium is composed of the following ingredients (per liter of distilled water): $0.3 \mathrm{~g}$ of $\mathrm{KH}_{2} \mathrm{PO}_{4} ; 1 \mathrm{~g}$ of $\mathrm{NH}_{4} \mathrm{Cl} ; 0.1 \mathrm{~g}$ of $\mathrm{MgCl}_{2} \cdot 7 \mathrm{H}_{2} \mathrm{O} ; 0.08 \mathrm{~g}$ of $\mathrm{CaCl}_{2} \cdot 7 \mathrm{H}_{2} \mathrm{O} ; 0.6 \mathrm{~g}$ of $\mathrm{NaCl} ; 2 \mathrm{~g}$ of $\mathrm{KHCO}_{3} ; 9.52 \mathrm{~g}$ of $4-(2-$ hydroxyethyl)-1-piperazine ethanesulfonic acid (HEPES); $1 \mathrm{~g}$ yeast extract; $1 \mathrm{~g}$ peptone; and $10 \mathrm{~mL}$ each of trace metal solution and vitamin solution (Sekiguchi et al., 2000). The $\mathrm{pH}$ of the medium was adjusted to 7.0 by adding $6 \mathrm{~N} \mathrm{KOH}$ solution. G. metallireducens was monocultured in IET-P medium in the presence of ethanol $(20 \mathrm{mM})$ as an electron donor and ferric citrate $(20 \mathrm{mM})$ as an electron acceptor, as reported previously (Rotaru et al., 2014a). M. barkeri was monocultured in IET-P medium supplemented with sodium acetate $(20 \mathrm{mM})$. Monocultures of either species were maintained by routinely transferring exponentially growing culture (ca. 5-10\% volume) to the fresh medium. To establish coculture, both species from exponentially growing monocultures were inoculated into IET-P medium containing ethanol $(20 \mathrm{mM})$ as the electron donor and various GO-based materials (see below). The initial cell 
density of G. metallireducens and M. barkeri was set at $2.5 \times 10^{6}$ cells $/ \mathrm{mL}$ and $2.5 \times 10^{5}$ cells $/ \mathrm{mL}$, respectively, unless otherwise mentioned. Condensed inoculum prepared by centrifugation under an anaerobic atmosphere was used, if necessary. Carbon dioxide and bicarbonate ion in the medium served as potential electron acceptors (Rotaru et al., 2014a). The inoculated culture bottle was purged with $\mathrm{N}_{2}+\mathrm{CO}_{2}$ (80:20) to remove carry-over methane from the inoculum and then cultivated as described above for 90 days. During cultivation, cultures were periodically sampled with 5- or 10-day intervals. All culture experiments were conducted in triplicate. Tukey's honest significance difference test was conducted for statistical analysis.

\section{Preparation of GO-Based Materials Amended Media}

All GO-based materials were purchased from NanoInnova Technologies SL (Toledo, Spain). Figure 1 shows model structures of the GO-based materials used in this study. GObased material was suspended in IET-P medium and vigorously mixed with a vortex mixer. The suspension was then centrifuged $\left(6,000 \times g, 20 \mathrm{~min}, 4^{\circ} \mathrm{C}\right)$ and resuspended in fresh IET-P medium for the following sonication process. This washing process was performed in order to remove possible inhibitory substances contained in the GO-based materials and to avoid the adsorption of trace elements that are necessary for microbial growth. Each GO-based material was suspended in IET-P medium at a concentration of $0.1 \%(\mathrm{w} / \mathrm{v})$. The suspension was then sonicated using a UD-201 Ultrasonic disruptor (TOMY, Tokyo, Japan) for 1 min with 5-s intervals on ice, and dispensed to the glass bottle for cultivation. This sonication process produced medium with homogeneously suspended GO-based material (Figure 2).

\section{Gas Phase Analysis}

For evaluating DIET efficiency, methane concentration in the gas phase was monitored periodically with a gas chromatograph GC-2014 (Shimadzu, Kyoto, Japan) equipped with a Rt-QPLOT column (30 m, 0.32 mm I.D, $10 \mu \mathrm{m}$ F.T.; Restek, Bellefonte, PA, United States) and flame ionization detector (FID) as described previously (Kato et al., 2015).

\section{Liquid Phase Analysis}

Liquid phase components, such as ethanol and acetate, were quantified throughout cultivation by using a high-performance liquid chromatography (HPLC) system (D-2000 LaChrom Elite HPLC system; Hitachi, Tokyo, Japan) equipped with Aminex ${ }^{\circledR}$ HPX-87H column (300 mm, 7.8 mm I.D.; Bio-Rad Laboratories, Hercules, CA, United States), a ultraviolet (UV) detector at $240 \mathrm{~nm}$ (L-2400, Hitachi, Tokyo, Japan), and refractive index (RI) (L-2490, Hitachi, Tokyo, Japan), as described previously (Kato et al., 2015). The liquid sample (ca. $150 \mu \mathrm{L}$ ) was periodically collected with a disposable syringe. The filtered sample $(10 \mu \mathrm{L})$ was directly injected into the HPLC circuit and chromatographed.

\section{Carbon Isotope Labeling Experiment}

${ }^{13} \mathrm{C}$-labeled sodium bicarbonate $\left(\mathrm{NaH}^{13} \mathrm{CO}_{3}\right)$ was supplemented to the coculture medium with $\mathrm{GO}$-amendment to obtain the final concentration of $0.5 \mathrm{mM}$. The culture bottle was cultivated as described above (section "Microorganisms and Cultivations"). Carbon isotopic compositions of methane and carbon dioxide in the gas phase of the culture bottle was determined, as previously described (Mayumi et al., 2016). In brief, gas-phase samples were taken at the initial time point of the cultivation and the stationary phase and then injected into sealed glass bottles in which the gas phase was vacuumed. Carbon isotopic compositions of methane and carbon dioxide in the gas samples were determined by gas chromatography-combustion isotope ratio mass spectrometry (GC-C-IRMS) consisting of a Trace GC Ultra, a GC IsoLink, a ConFlo IV, and a DELTA V Plus IRMS system (Thermo Fisher Scientific Inc., Waltham, MA, United States). The isotopic data were obtained as $\delta$ values in the IRMS analysis and converted to atm\% by calculating $(1+\delta / 1000) \times 1.124$. The ratio of $\mathrm{CO}_{2}$-reduction derived methane was calculated by the method described elsewhere (Hori et al., 2011).

\section{Quantification of GO-Based Materials-Associated Cells}

The number of cells attached to the GO-based material surface was quantified by measuring the decrease in planktonic cell density, as described below. G. metallireducens and/or M. barkeri

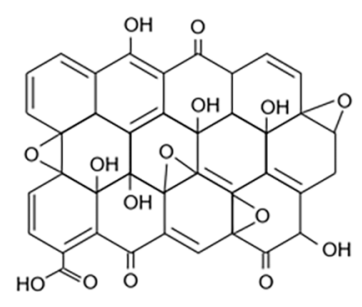

$\mathrm{GO}$

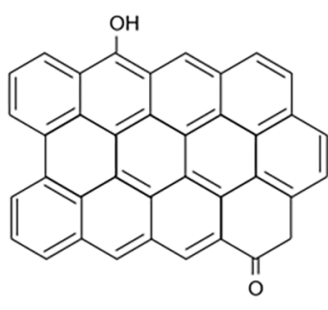

rGO

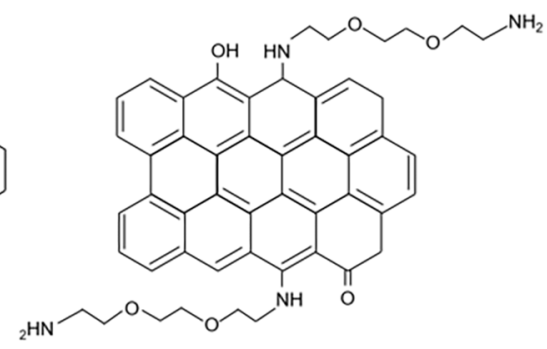

rGO-NH $\mathrm{N}_{2}$

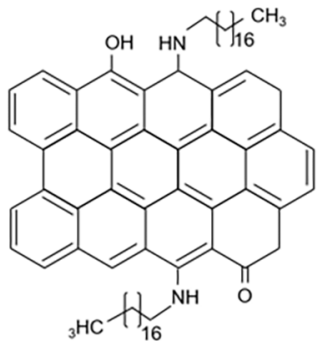

rGO-ODA

FIGURE 1 | Model structures of GO-based materials used in this study. 


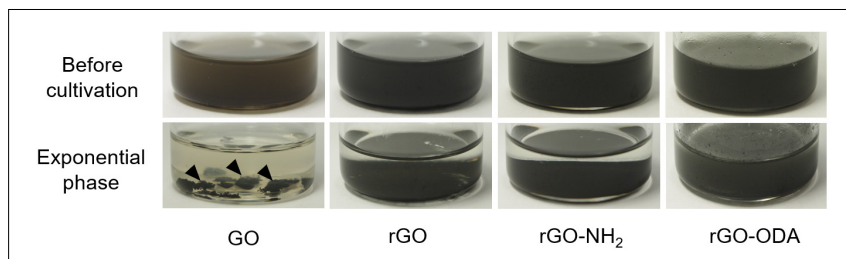

FIGURE 2 | The appearance of coculture bottles containing different GO-based materials before cultivation and the exponential phase. Arrowheads indicate self-aggregated hydrogels.

from the exponential phase monoculture were inoculated in IET$\mathrm{P}$ medium containing ethanol $(20 \mathrm{mM})$ and each $\mathrm{GO}$-based material under the same conditions of coculture experiment as described above (section "Microorganisms and Cultivations"), except the initial cell density of G. metallireducens and M. barkeri was set to $2.5 \times 10^{8}$ cells $/ \mathrm{mL}$ and $2.5 \times 10^{7}$ cells $/ \mathrm{mL}$, respectively, in order to obtain significant cell densities through a direct counting method. During cultivation $(0-250 \mathrm{~h}), 100 \mu \mathrm{L}$ of culture was periodically sampled with a disposable syringe. The sample was transferred to a microcentrifuge tube and centrifuged $\left(100 \times g, 3 \mathrm{~min}, 4^{\circ} \mathrm{C}\right)$ to separate planktonic cells from GO-based materials-associated cells. The liquid phase containing planktonic cells was mixed with a LIVE/DEAD BacLight ${ }^{\mathrm{TM}}$ Bacterial Viability Kit (LIVE/DEAD; Molecular Probes, Eugene, OR, United States) as described previously (Igarashi and Kuwabara, 2014). Cell density was determined by direct counting using a bacterial counting chamber and an optical microscope. The total number of planktonic cells was determined by counting under blue light excitation. During coculture, G. metallireducens and $M$. barkeri were distinguished by their morphology and based on $\mathrm{F}_{420}$ autofluorescence under violet light excitation, as reported previously (Igarashi and Kuwabara, 2014).

\section{X-Ray Photoelectron Spectroscopy (XPS)}

Chemical states of GO-based materials were analyzed by XPS. Sample for XPS was collected from the culture medium by centrifugation $\left(1,000 \times g, 5 \mathrm{~min}, 4^{\circ} \mathrm{C}\right)$ and processed to make an XPS specimen, as described previously (Salas et al., 2010). In brief, each GO-based materials sample was rinsed with MilliQ water three times, then washed with $80 \%$ ethanol (3-5 min), Milli-Q water (1 min), $1 \mathrm{~N} \mathrm{HCl} \mathrm{(3-5} \mathrm{min),} \mathrm{and} \mathrm{rinsed} \mathrm{with}$ Milli-Q water at least three times. This sample was air-dried and stored in a desiccator. For the preparation of XPS specimen, the dried sample was resuspended in Milli-Q water, dispersed onto an Al plate, and dried under vacuum. XPS spectrum of C1s was obtained at the scan ranges of 275-300 eV using a PHI5000Versa Probe (ULVAC-PHI, Inc., Kanagawa, Japan) equipped with a monochromatic $\mathrm{Al} \mathrm{K}_{\alpha} \mathrm{X}$-ray source. Purchased GO and $\mathrm{rGO}$ were analyzed and referenced as standards.

\section{Field-Emission Scanning Electron Microscopy (FE-SEM)}

Field-Emission Scanning Electron Microscopy sample containing GO-based materials was collected from the exponentially growing culture (methane concentration ranged in $10-15 \mathrm{mM})$ and fixed with $2 \%(\mathrm{w} / \mathrm{v})$ glutaraldehyde in $0.1 \mathrm{M}$ sodium cacodylate buffer $(\mathrm{pH} 7.2$ ) for $2 \mathrm{~h}$ at room temperature, and processed as described previously (Igarashi et al., 2016). Prepared FE-SEM specimen was mounted on an aluminum stab using carbon tape. The specimen was coated with platinum/palladium alloy with an ion sputter E102 (Hitachi, Tokyo, Japan) and observed with an FE-SEM (JSM$6330 \mathrm{~F}$ or JSM-7800F; JEOL, Tokyo, Japan) at an acceleration voltage of $5 \mathrm{kV}$.

\section{RESULTS}

\section{Growth via GO-Mediated DIET}

We examined the DIET-promoting efficiency of GO on model coculture of $G$. metallireducens and M. barkeri. Amendment of GO induced methane production and ethanol consumption (Figures 3A,B), while coculture without GO (Figure 3) or monocultures of either microorganism in the presence of GO (Supplementary Figure S1) showed no methane production during the cultivation period tested (Figure 3). In the GOamended coculture, acetate, a product of ethanol oxidation by G. metallireducens, was transiently accumulated in DIETmediated ethanol degradation at the early growth phase and was nearly completely consumed during cultivation (Figure 3C). Such complete degradation of ethanol is possible only when syntrophic ethanol oxidation occurs. Theoretically, each mole of ethanol in the coculture should be converted to $1.5 \mathrm{~mol}$ of methane, according to the reaction schemes showing step-wise degradation of ethanol (Rotaru et al., 2014a).

Ethanol oxidation to acetate:

$$
2 \mathrm{C}_{2} \mathrm{H}_{5} \mathrm{OH}+2 \mathrm{H}_{2} \mathrm{O} \rightarrow 2 \mathrm{CH}_{3} \mathrm{COOH}+8 \mathrm{H}^{+}+8 \mathrm{e}^{-}
$$

$\mathrm{CH}_{4}$ production by acetate disproportion:

$$
2 \mathrm{CH}_{3} \mathrm{COOH} \rightarrow 2 \mathrm{CH}_{4}+2 \mathrm{CO}_{2}
$$

$\mathrm{CH}_{4}$ production by DIET-mediated $\mathrm{CO}_{2}$ reduction:

$$
\mathrm{CO}_{2}+8 \mathrm{H}^{+}+8 \mathrm{e}^{-} \rightarrow \mathrm{CH}_{4}+2 \mathrm{H}_{2} \mathrm{O}
$$

Overall reaction (Eqs. $1+2+3)$ :

$$
2 \mathrm{C}_{2} \mathrm{H}_{5} \mathrm{OH} \rightarrow 3 \mathrm{CH}_{4}+\mathrm{CO}_{2}
$$

The GO-amended coculture produced around $248 \mu \mathrm{mol}$ methane from $210 \mu \mathrm{mol}$ ethanol (Figures 3A,B), which corresponded to a conversion ratio of ca. 1.2. Although the conversion ratio is slightly smaller than the expected ratio (1.5), part of the electrons generated from ethanol oxidation was expectedly consumed for GO reduction. The amount of electrons potentially consumed by GO reduction was assessed in the pure culture of G. metallireducens supplemented with ethanol and GO (Supplementary Figure S1A). G. metallireducens consumed around $65 \mu \mathrm{mol}$ ethanol for the reduction of GO. This ethanol consumption is equal to ca. $260 \mu \mathrm{mol}$ electron consumption (Eq. 1), and thus expected decrease in methane is ca. $33 \mu \mathrm{mol}$ (Eq. 3), which corresponds to $89 \%$ 

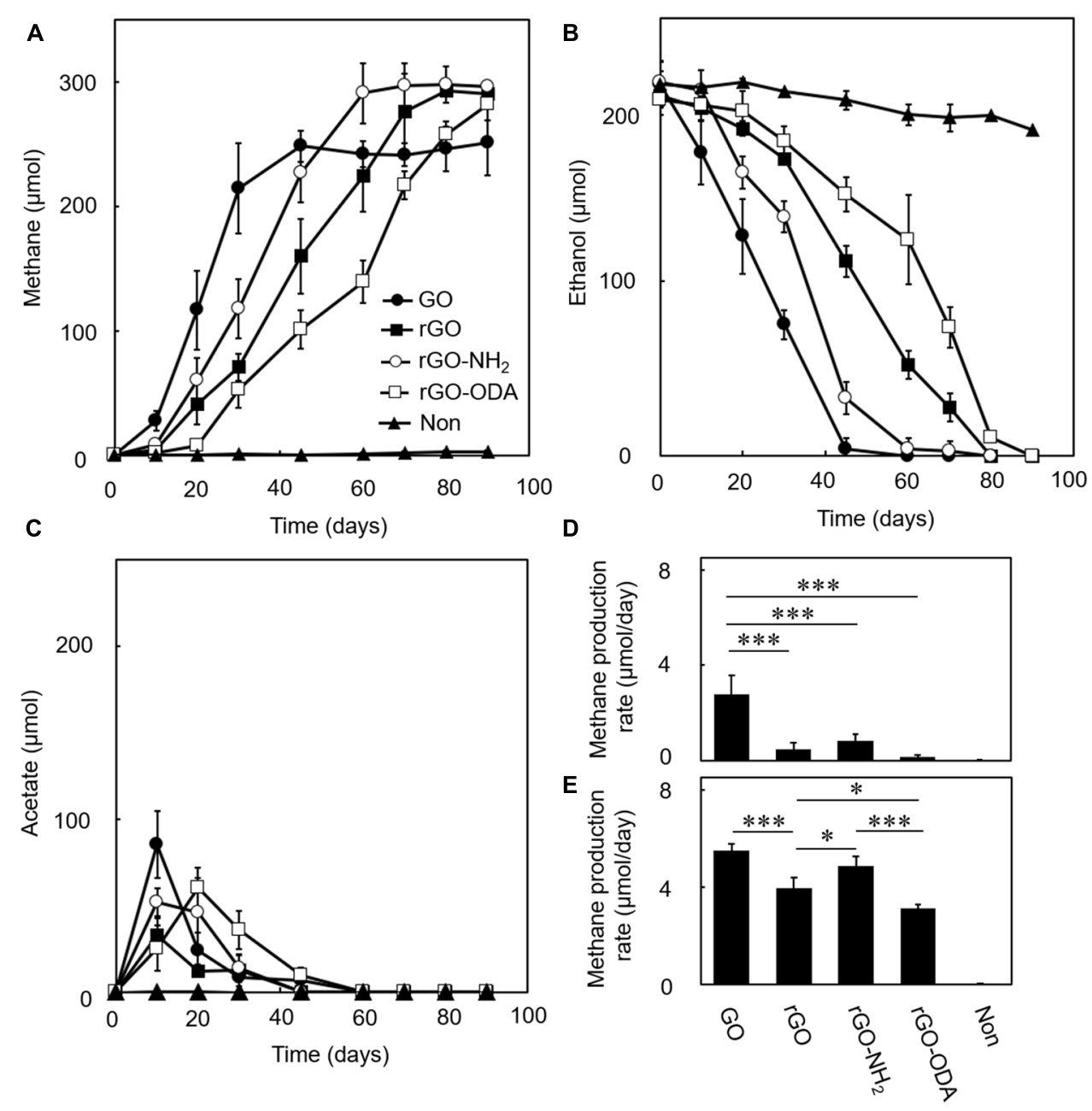

GO-based materials

FIGURE 3 | Difference in DIET-promoting efficiencies for GO-based materials. DIET cocultures of Geobacter metallireducens and Methanosarcina barkeri were conducted in the presence of each GO-based material using ethanol as the substrate. Methane (A), ethanol (B), and acetate (C) amounts were measured periodically during cultivations. Methane production rates in early- (D) and mid-exponential (E) phases were calculated from the data in (A). Data are presented as the means of three independent cultures, and error bars represent standard deviations. ${ }^{* * *} p<0.001,{ }^{*} p<0.05$ using one-way analysis of variance with Tukey's honest significance difference test.

of the observed decrease in methane (ca. $38 \mu \mathrm{mol}$ ) in GOamended coculture. The remaining shortage was expectedly utilized for cell growth. These observations suggest that DIETmediated syntrophic methanogenesis occurred in the GOamended coculture.

In order to obtain further evidence for the occurrence of DIET-mediated syntrophic methanogenesis, we examined the carbon flux of the coculture by isotope labeling experiment. If ethanol is completely degraded via DIET-mediated syntrophic methanogenesis (Eq. 4), one-third of methane derives from $\mathrm{CO}_{2}$ reduction and the other two thirds from acetate disproportionation (acetoclastic methanogenesis). The GOamended coculture with the supplementation of ${ }^{13} \mathrm{CO}_{2}$ showed that $31 \pm 9.7 \%$ of methane was produced by $\mathrm{CO}_{2}$ reduction, which was comparable to the expected value. This result suggests that methane production in the GO-amended coculture occurred as the result of DIET-dependent $\mathrm{CO}_{2}$ reduction and DIET-independent acetate disproportionation.

Graphene oxide-amended coculture exhibited a change in color, where the suspension changed from brown to black and formed self-aggregated hydrogels from the early exponential phase to the end of cultivation (Figure 2). The hydrogels were also observed in the GO-amended monoculture of G. metallireducens. Such hydrogels have been reported in monocultures of Geobacter species (Yoshida et al., 2016b) as well as in the reaction product resulting from an abiotic reduction of GO (Xu et al., 2010). Thus, it is highly probable that $G$. metallireducens in this study reduced oxygen-containing functional groups on the GO surface and induced the formation of the hydrogel.

In order to determine the biological reduction of GO during cultivation, XPS analysis was carried out for GO collected from 
exponentially growing cultures. Figure 4 summarizes the XPS spectra of GO specimens before and after cultivation. Spectrum from $\mathrm{GO}$ confirmed the presence of peaks for the $\mathrm{C}-\mathrm{OH}(286 \mathrm{eV})$ and $C=\mathrm{O}(288 \mathrm{eV})$ bonds (Wang et al., 2011), suggesting that GO surface is highly abundant in oxygen-containing functional groups (Szabó et al., 2005). After cocultivation, these peaks were dramatically decreased, and the peak derived from the C-C bond (284-285) (Yang et al., 2009) appeared on the specimen (Figure 4). This spectrum change was also observed on GO monocultured with G. metallireducens. No spectrum change was observed for GO incubated without microorganism. These results conclusively suggest that G. metallireducens mainly contributes to the reduction of GO to rGO in coculture and also induces self-aggregation of biologically reduced GO shown in Figure 2. Collectively, these results demonstrate that biologically reduced GO can promote DIET, acting as a conduit connecting electroactive cells.

\section{Effects of Surface Modification of rGO}

Next, we attempted to elucidate the effects of rGO surface functional groups on DIET-promoting efficiency. For the comparison, chemically reduced GO, a hydrophilic amine-functionalized $\mathrm{rGO}\left(\mathrm{rGO}-\mathrm{NH}_{2}\right)$, and hydrophobic octadecylamine-functionalized rGO (rGO-ODA) were employed as model DIET-promoting GO-based materials. Cocultures with $\mathrm{rGO}$ and $\mathrm{rGO}-\mathrm{NH}_{2}$ exhibited a stiff layer of sediment at the bottom of the culture bottle. However, coculture bottles amended with rGO-ODA did not show these macroscopic changes (Figure 2, bottom panels).

Reduced GO and its derivatives induced methane production with a near-stoichiometric ratio of 1.5 (Figure 3A), whereas monocultures with rGO did not induce methane production (Supplementary Figure S1). The DIET-promoting efficiencies of the three rGO derivatives were evaluated by methane production rates in the early- (Figure 3D) and mid-exponential phases (Figure 3E). $\mathrm{rGO}-\mathrm{NH}_{2}$ showed the highest methane production rate among GO-based materials tested, which is followed by rGO

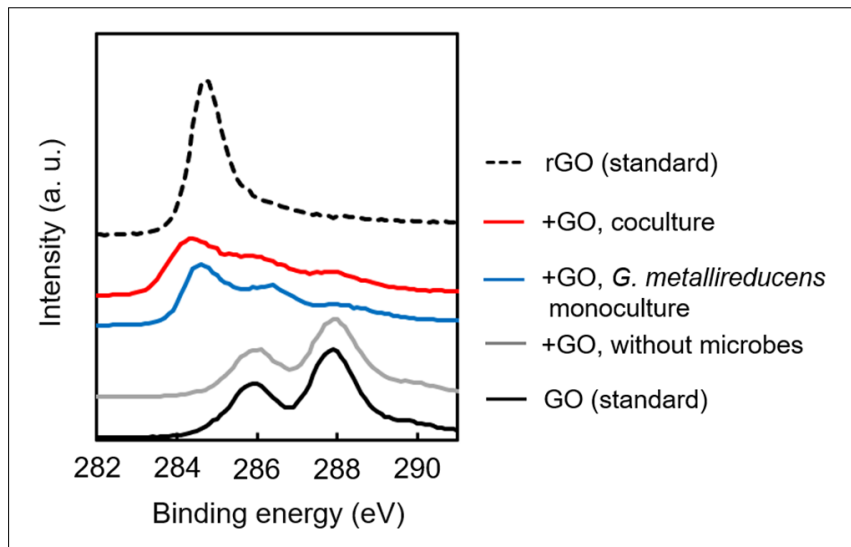

FIGURE 4 | X-ray Photoelectron Spectroscopy spectra of GO-based materials. Dried specimens of GO-based materials collected from culture media before and after cultivation were analyzed by XPS. Binding energy of 284-285, C-C bond; 286, C-OH bond; 288, C=O bond. and $\mathrm{rGO}-\mathrm{ODA}$. This result suggests that surface hydrophilicity is positively correlated with DIET-promoting efficiency.

\section{Fine Structures of GO-Based Material Surface}

In order to examine the fine structures of GO-based materials, surface morphologies of GO-based materials derived from exponentially growing cultures were observed under FE-SEM (Figure 5). The surface of GO and $\mathrm{rGO}-\mathrm{NH}_{2}$ was enriched for both $G$. metallireducens and M. barkeri. In the specimens isolated from $\mathrm{GO}$, rGO, and rGO- $\mathrm{NH}_{2}$ cocultures, cells were sometimes found to be embedded in exopolysaccharide (EPS)-like structures (Figures 5A-C; Dohnalkova et al., 2011), indicating that these microorganisms actively grew and colonized the GO-based material surface. EPS production is thought to contribute to the self-aggregation of rGO hydrogels and also to cause stiff sediment, as was observed in the rGO and $\mathrm{rGO}-\mathrm{NH}_{2}$ coculture (Figure 2). Although $\mathrm{GO}$ has been reduced to $\mathrm{rGO}$ during cultivation (Figure 4) no significant change in surface morphology of cultivated GO was observed after cultivation as compared to before cultivation (Figures 5A,E).

\section{Measurement of Cell Attachment to GO-Based Materials}

Considering these results, we hypothesized that attachment of cells to GO-based materials during the initial stage of cultivation might strongly affect DIET-promoting efficiency. Hence, we determined attachment efficiency by mixing microbial cells and each GO-based material and measuring the decrease in planktonic cell densities. The density of planktonic cells in GOamended monoculture of $G$. metallireducens rapidly decreased within $50 \mathrm{~h}$ of cultivation, followed by $\mathrm{rGO}-\mathrm{NH}_{2-}$, rGO-, and rGO-ODA-amended cultures, respectively (Figure 6A). This trend was also observed for the cocultures (Figure 6B). These differences in cell attachment rate reflect differences in DIETpromoting efficiency (Figure 3). In the case of $M$. barkeri, no significant change in GO-based material attachment rate was observed in monoculture (Figure 6C), probably because M. barkeri cannot be metabolically active in this culture condition. However, coculture with G. metallireducens in the presence of GO and rGO- $\mathrm{NH}_{2}$ showed a noticeable decrease in planktonic $M$. barkeri cell density (Figure 6D). These results indicate that attachment of $M$. barkeri to GO-based materials

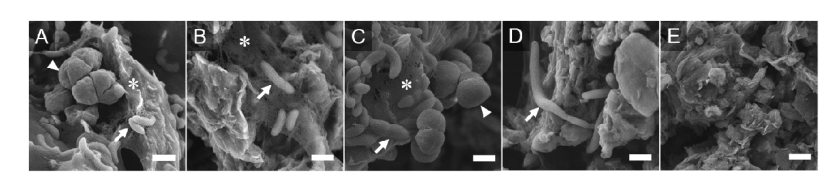

FIGURE 5 | Field-Emission Scanning Electron Microscopy images of GO-based materials surfaces. Representative images of GO-based material surface morphology of exponentially growing cocultures are shown. Arrows indicate G. metallireducens. Arrowheads indicate M. barkeri. GO (A); rGO (B); rGO-NH $\mathrm{N}_{2}$ (C); rGO-ODA (D); GO before cultivation (E). Bars, $1 \mu \mathrm{m}$.

*Exopolysaccharide-like structure. 

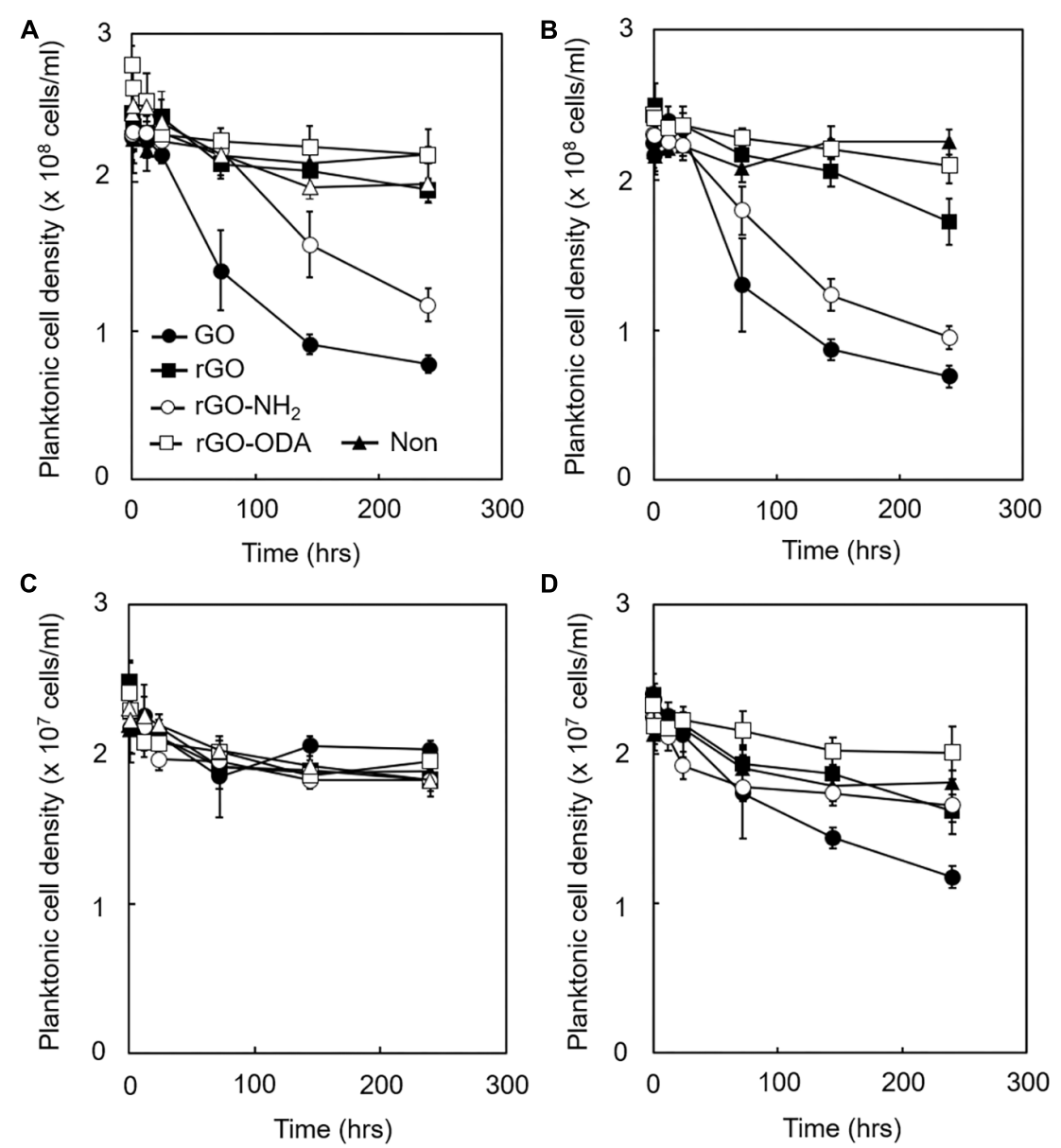

FIGURE 6 | Adhesion efficiency of microbial cells to GO-based materials. Each GO-based material was supplemented with IET-P medium containing ethanol (20 mM). The planktonic cell density of $\mathbf{G}$. metallireducens (A,B) and M. barkeri (C,D) is shown. Monoculture (A,C). Coculture (B,D). Cultivations were conducted under the same cultivation conditions as described in section "Microorganisms and Cultivations," except the initial cell density of G. metallireducens and $M$. barkeri was set to $2.5 \times 10^{8} \mathrm{cells} / \mathrm{mL}$ and $2.5 \times 10^{7}$ cells $/ \mathrm{mL}$, respectively. Data are presented as the means of three independent cultures, and error bars represent standard deviations.

was strongly controlled by its DIET-partner (G. metallireducens), possibly via alteration of GO-based material surface properties due to surface reduction and subsequent formation of biofilm containing EPS. Most populations (>90\%) of planktonic cells for microorganisms both in monoculture and coculture were viable under LIVE/DEAD observation during the entire cultivation period ( $250 \mathrm{~h}$, data not shown). This observation indicates that the antimicrobial activity of GO-based materials (Hu et al., 2010) was negligible in our cultures, and thus the decrease we observed in planktonic cell density was not due to cell lysis, but was a result of adhesion to GO-based materials.

\section{DISCUSSION}

In this study, we provide clear evidence that biologically and chemically reduced GO promotes methane production by a defined model coculture of $G$. metallireducens and M. barkeri. The stoichiometry of methane production from ethanol and the isotope labeling experiments suggests that DIET-mediated syntrophic methanogenesis occurred in the cocultures amended with GO-based materials. This study also showed that the surface properties of GO-based materials, in particular, their hydrophilicity, significantly affect their DIET-promoting efficiency. G. metallireducens cells were quickly recruited onto hydrophilic GO-based materials (especially GO) before the establishment of DIET (Figure 6). It can be assumed that GO has physicochemical properties allowing for efficient recruitment and enrichment for Geobacter species. This assumption is supported by a previous report demonstrating selective enrichment of Geobacter species on GO (Yoshida et al., 2016a,b). Adhesion of M. barkeri was promoted in the presence of G. metallireducens (Figures 6C,D), suggesting that G. metallireducens formed environments where $M$. barkeri can establish strong affinity to the GO-based material surface probably via EPS (Figure 5) and promote DIET-dependent methane production. Considering that the GO-amended coculture showed significantly higher methanogenic rate in 
the early phase of syntrophic methanogenesis than other rGO derivatives (Figure 3D), attachment of G. metallireducens, followed by $M$. barkeri, onto the GO-based materials would be the rate-limiting step for initiation of the DIET-dependent syntrophic methanogenesis. Our results suggest that the DIET-promoting efficiency can be modulated by the addition of oxygen-containing functional groups on the GO-based material surface, while further detailed examination, including measurements of the surface hydrophilicity and in situ conductivity of GO-based materials, are required to evaluate the DIET-promoting efficiency quantitatively. This insight can also be applied to a wide range of conductive materials, which have been reported to enhance DIET of various microbial communities.

This study assessed DIET-promoting efficiency using a representative model DIET coculture. Hence, further experiments using different Geobacter species (Kato, 2017) and/or methanogens may provide additional data supporting a comprehensive understanding of the physical interaction of solid materials and microbial cells. We suspect that culture conditions also affect redox potential and surface charge of conductive materials. Detailed electrochemical analysis of conductive materials for each culture condition will help to identify the key factor(s) that specifically control the efficiency of DIET (Liu et al., 2010). In addition, metatranscriptome analysis for model DIET cultures may provide clues to elucidate mechanisms whereby electroactive microorganisms sense and attach to solid materials with different physicochemical properties.

From a practical point of view, the selection of proper materials for the promotion of DIET is crucial to establish efficient and robust DIET systems for industrial level implementation (Sasaki et al., 2018). This study showed that GO is the superior solid material for DIET promotion than other GO-based materials (Figure 3). Furthermore, we have also performed the similar coculture experiments using magnetite, that have frequently been used as a conductive material to promote DIET (Kato et al., 2012a,b; Baek et al., 2015; Yamada et al., 2015; Yin et al., 2016), instead of the GO-based materials. Methane production rate of the GO-amended coculture in the mid-exponential phase $(5.5 \pm 0.3 \mu \mathrm{mol} / \mathrm{day}$, Figure $3 \mathrm{E})$ was significantly higher than that of the magnetite-amended coculture $(3.1 \pm 0.2 \mu \mathrm{mol} / \mathrm{day})$, suggesting that $\mathrm{GO}$ is the superior material for DIET promotion than conventionally utilized conductive materials. Although GO has been bulkproduced in industrial level already, large-scale utilization of GO and its derivatives in engineered environments would still be prohibited by the low cost-effectiveness. However, GObased materials are suitable for experimentally investigating

\section{REFERENCES}

Baek, G., Kim, J., Cho, K., Bae, H., and Lee, C. (2015). The biostimulation of anaerobic digestion with (semi)conductive ferric oxides: their potential for enhanced biomethanation. Appl. Microbiol. Biotechnol. 99, 10355-10366. doi: 10.1007/s00253-015-6900-y

Chen, S., Rotaru, A.-E., Liu, F., Philips, J., Woodard, T. L., Nevin, K. P., et al. (2014a). Carbon cloth stimulates direct interspecies electron transfer in the mechanisms for DIET promotion, because it is relatively easy to modify their chemical properties by adding various functional groups (Li et al., 2014). Results obtained in this study provide fundamental knowledge for designing and selection of suitable solid materials that efficiently promote DIET. Fabrication of functionalized solids materials from cheaper solids such as iron oxides and activated carbon, which have similar physicochemical properties of GO, would increase costeffectiveness. Furthermore, fundamental analysis using model DIET cocultures combined with an examination of pilot-scale DIET systems (Kumar et al., 2014; Zhou et al., 2019) will further promote industrial applications of DIET.

\section{DATA AVAILABILITY STATEMENT}

All datasets generated for this study are included in the article/Supplementary Material.

\section{AUTHOR CONTRIBUTIONS}

KI and SK designed the experiments. KI performed the experiments and wrote the initial draft of the manuscript. All authors interpreted the data and contributed to the editing of the manuscript.

\section{FUNDING}

This research was supported by the Japan Society for the Promotion of Science, KAKENHI Grant Number 16H06191.

\section{ACKNOWLEDGMENTS}

We thank Dr. Kawaguchi Kenji for technical help with XPS analysis. We also thank Dr. Mayumi Daisuke for help in carbon isotope measurement. This study was supported by the NIMS Nanofabrication Platform in Nanotechnology Platform Project sponsored by the Ministry of Education, Culture, Sports, Science and Technology (MEXT), Japan.

\section{SUPPLEMENTARY MATERIAL}

The Supplementary Material for this article can be found online at: https://www.frontiersin.org/articles/10.3389/fmicb. 2019.03068/full\#supplementary-material

syntrophic co-cultures. Bioresour. Technol. 173, 82-86. doi: 10.1016/j.biortech. 2014.09.009

Chen, S., Rotaru, A.-E., Shrestha, P. M., Malvankar, N. S., Liu, F., Fan, W., et al. (2014b). Promoting interspecies electron transfer with biochar. Sci. Rep. 4:5019.

Dang, Y., Holmes, D. E., Zhao, Z., Woodard, T. L., Zhang, Y., Sun, D., et al. (2016). Enhancing anaerobic digestion of complex organic waste with carbon-based conductive materials. Bioresour. Technol. 220, 516-522. doi: 10.1016/j.biortech. 2016.08.114 
Dohnalkova, A. C., Marshall, M. J., Arey, B. W., Williams, K. H., Buck, E. C., and Fredrickson, J. K. (2011). Imaging hydrated microbial extracellular polymers: comparative analysis by electron microscopy. Appl. Environ. Microbiol. 77, 1254-1262. doi: 10.1128/AEM.02001-10

Goto, Y., and Yoshida, N. (2016). Preliminary evaluation of a microbial fuel cell treating artificial dialysis wastewater using graphene oxide. AIP Conf. Proc. 1709:020007.

Hori, T., Sasaki, D., Haruta, S., Shigematsu, T., Ueno, Y., Ishii, M., et al. (2011). Detection of active, potentially acetate-oxidizing syntrophs in an anaerobic digester by flux measurement and formyltetrahydrofolate synthetase (FTHFS) expression profiling. Microbiology 157, 1980-1989. doi: 10.1099/mic.0.049 189-0

Hu, W., Peng, C., Luo, W., Lv, M., Li, X., Li, D., et al. (2010). Graphene-based antibacterial paper. ACS Nano. 4, 4317-4323. doi: 10.1021/nn101097v

Hummers, W. S., and Offeman, R. E. (1958). Preparation of graphitic oxide. J. Am. Chem. Soc. 80, 1339-1339. doi: 10.1021/ja01539a017

Igarashi, K., and Kuwabara, T. (2014). Fe(III) oxides protect fermentermethanogen syntrophy against interruption by elemental sulfur via stiffening of $\mathrm{Fe}$ (II) sulfides produced by sulfur respiration. Extremophiles 18, 351-361. doi: 10.1007/s00792-013-0620-y

Igarashi, K., Yamamura, Y., and Kuwabara, T. (2016). Natural synthesis of bioactive greigite by solid-gas reactions. Geochim. Cosmochim. Acta 191, 47-57. doi: 10.1016/j.gca.2016.07.005

Jiao, Y., Qian, F., Li, Y., Wang, G., Saltikov, C. W., and Gralnick, J. A. (2011). Deciphering the electron transport pathway for graphene oxide reduction by Shewanella oneidensis MR-1. J. Bacteriol. 193, 3662-3665. doi: 10.1128/JB. 00201-11

Kamran, U., Heo, Y. J., Lee, J. W., and Park, S. J. (2019). Functionalized carbon materials for electronic devices: a review. Micromachines 10, E234. doi: 10.3390/ mil0040234

Kato, S. (2015). Biotechnological aspects of microbial extracellular electron transfer. Microbes Environ. 30, 133-139. doi: 10.1264/jsme2.ME15028

Kato, S. (2017). Influence of anode potentials on current generation and extracellular electron transfer paths of Geobacter species. Int. J. Mol. Sci. 18:E108. doi: 10.3390/ijms18010108

Kato, S., Hashimoto, K., and Watanabe, K. (2012a). Methanogenesis facilitated by electric syntrophy via (semi)conductive iron-oxide minerals. Environ. Microbiol. 14, 1646-1654. doi: 10.1111/j.1462-2920.2011.02611.x

Kato, S., Hashimoto, K., and Watanabe, K. (2012b). Microbial interspecies electron transfer via electric currents through conductive minerals. Proc. Natl. Acad. Sci. U.S.A. 109, 10042-10046. doi: 10.1073/pnas.1117592109

Kato, S., Yumoto, I., and Kamagata, Y. (2015). Isolation of acetogenic bacteria that induce biocorrosion by utilizing metallic iron as the sole electron donor. Appl. Environ. Microbiol. 81, 67-73. doi: 10.1128/AEM.02767-14

Kostarelos, K., and Novoselov, K. S. (2014). Exploring the interface of graphene and biology. Science 344, 261-263. doi: 10.1126/science.1246736

Kouzuma, A., Kato, S., and Watanabe, K. (2015). Microbial interspecies interactions: recent findings in syntrophic consortia. Front. Microbiol. 6:477. doi: $10.3389 /$ fmicb. 2015.00477

Kumar, G. G., Kirubaharan, C. J., Udhayakumar, S., Ramachandran, K., Karthikeyan, C., Renganathan, R., et al. (2014). Synthesis, structural, and morphological characterizations of reduced graphene oxide-supported polypyrrole anode catalysts for improved microbial fuel cell performances. ACS Sustainable Chem. Eng. 2, 2283-2290. doi: 10.1021/sc500244f

Lee, J. Y., Lee, S. H., and Park, H. D. (2016). Enrichment of specific electro-active microorganisms and enhancement of methane production by adding granular activated carbon in anaerobic reactors. Bioresour. Technol. 205, 205-212. doi: 10.1016/j.biortech.2016.01.054

Li, J., Zeng, X., Ren, T., and van der Heide, E. (2014). The preparation of graphene oxide and its derivatives and their application in bio-tribological systems. Lubricants 2, 137-161. doi: 10.3390/lubricants2030137

Li, L. L., Tong, Z. H., Fang, C. Y., Chu, J., and Yu, H. Q. (2015). Response of anaerobic granular sludge to single-wall carbon nanotube exposure. Water Res. 70, 1-8. doi: 10.1016/j.watres.2014.11.042

Lin, R., Cheng, J., Zhang, J., Zhou, J., Cen, K., and Murphy, J. D. (2017). Boosting biomethane yield and production rate with graphene: the potential of direct interspecies electron transfer in anaerobic digestion. Bioresour. Technol. 239, 345-352. doi: 10.1016/j.biortech.2017.05.017
Liu, F., Rotaru, A.-E., Shrestha, P. M., Malvankar, N. S., Nevin, K. P., and Lovley, D. R. (2012). Promoting direct interspecies electron transfer with activated carbon. Energy Environ. Sci. 5, 8982-8989.

Liu, H., Matsuda, S., Kato, S., Hashimoto, K., and Nakanishi, S. (2010). Redoxresponsive switching in bacterial respiratory pathway involving extracellular electron transfer. Chem. Sus. Chem. 3, 1253-1256. doi: 10.1002/cssc.20100 0213

Lovley, D. R. (2011). Reach out and touch someone: potential impact of DIET (direct interspecies energy transfer) on anaerobic biogeochemistry, bioremediation, and bioenergy. Rev. Environ. Sci. Biotechnol. 10, 101-105. doi: 10.1007/s11157-011-9236-9

Mayumi, D., Mochimaru, H., Tamaki, H., Yamamoto, K., Yoshioka, H., Suzuki, Y., et al. (2016). Methane production from coal by a single methanogen. Science $354,222-225$.

Novoselov, K. S., Geim, A. K., Morozov, S. V., Jiang, D., Zhang, Y., Dubonos, S. V., et al. (2004). Electric field effect in atomically thin carbon films. Science 306, 666-669. doi: 10.1126/science.1102896

Reguera, G., McCarthy, K. D., Mehta, T., Nicoll, J., Tuominen, M. T., and Lovley, D. R. (2005). Extracellular electron transfer via microbial nanowires. Nature 435, 1098-1101. doi: 10.1038/nature03661

Rotaru, A.-E., Shrestha, P. M., Liu, F., Markovaite, B., Chen, S., Nevin, K., et al. (2014a). Direct interspecies electron transfer between Geobacter metallireducens and Methanosarcina barkeri. Appl. Environ. Microbiol. 80, 4599-4605. doi: 10.1128/aem.00895-14

Rotaru, A.-E., Shrestha, P. M., Liu, F., Shrestha, M., Shrestha, D., Embree, M., et al. (2014b). A new model for electron flow during anaerobic digestion: direct interspecies electron transfer to Methanosaeta for the reduction of carbon dioxide to methane. Energy Environ. Sci. 7, 408-415. doi: 10.1039/c3ee42 $189 \mathrm{a}$

Salas, E. C., Sun, Z., Lüttge, A., and Tour, J. M. (2010). Reduction of graphene oxide via bacterial respiration. ACS Nano 4, 4852-4856. doi: 10.1021/nn101 $081 \mathrm{t}$

Sang, M., Shin, J., Kim, K., and Yu, K. J. (2019). Electronic and thermal properties of graphene and recent advances in graphene based electronics applications. Nanomaterials 9:E374. doi: 10.3390/nano9030374

Sasaki, K., Sasaki, D., Kamiya, K., Nakanishi, S., Kondo, A., and Kato, S. (2018). Electrochemical biotechnologies minimizing the required electrode assemblies. Curr. Opin. Biotechnol. 50, 182-188. doi: 10.1016/j.copbio.2018.0 1.016

Sekiguchi, Y., Kamagata, Y., Nakamura, K., Ohashi, A., and Harada, H. (2000). Syntrophothermus lipocalidus gen. nov., sp. nov., a novel thermophilic, syntrophic, fatty-acid-oxidizing anaerobe which utilizes isobutyrate. Int. J. Syst. Evol. Microbiol. 50, 771-779. doi: 10.1099/00207713-50-2-771

Summers, Z. M., Fogarty, H. E., Leang, C., Franks, A. E., Malvankar, N. S., and Lovley, D. R. (2010). Direct exchange of electrons within aggregates of an evolved syntrophic coculture of anaerobic bacteria. Science 330, 1413-1415. doi: 10.1126/science. 1196526

Szabó, T., Berkesi, O., and Dékány, I. (2005). DRIFT study of deuterium-exchanged graphite oxide. Carbon 43, 3186-3189. doi: 10.1016/j.carbon.2005.07.013

Tian, T., Qiao, S., Li, X., Zhang, M., and Zhou, J. (2017). Nano-graphene induced positive effects on methanogenesis in anaerobic digestion. Bioresour. Technol. 224, 41-47. doi: 10.1016/j.biortech.2016.10.058

Wang, G., Qian, F., Saltikov, C. W., Jiao, Y., and Li, Y. (2011). Microbial reduction of graphene oxide by Shewanella. Nano Res. 4, 563-570. doi: 10.1007/s12274011-0112-2

Xu, Y., Sheng, K., Li, C., and Shi, G. (2010). Self-assembled graphene hydrogel via a one-step hydrothermal process. ACS Nano 4, 4324-4330. doi: 10.1021/ nn101187z

Yamada, C., Kato, S., Ueno, Y., Ishii, M., and Igarashi, Y. (2015). Conductive iron oxides accelerate thermophilic methanogenesis from acetate and propionate. J. Biosci. Bioeng. 119, 678-682. doi: 10.1016/j.jbiosc.2014.11.001

Yang, D., Velamakanni, A., Bozoklu, G., Park, S., Stoller, M., Piner, R. D., et al. (2009). Chemical analysis of graphene oxide films after heat and chemical treatments by X-ray photoelectron and micro-raman spectroscopy. Carbon 47, 145-152. doi: 10.1016/j.carbon.2008.09.045

Yin, Q., Miao, J., Li, B., and Wu, G. (2016). Enhancing electron transfer by ferroferric oxide during the anaerobic treatment of synthetic wastewater with 
mixed organic carbon. Int. Biodeterior. Biodegrad. 119, 104-110. doi: 10.1016/j. ibiod.2016.09.023

Yong, Y. C., Yu, Y. Y., Zhang, X., and Song, H. (2014). Highly active bidirectional electron transfer by a self-assembled electroactive reduced graphene oxide hybridized biofilm. Angew. Chem., Int. Ed. 53, 4480-4483. doi: 10.1002/anie. 201400463

Yoshida, N., Goto, Y., and Miyata, Y. (2016a). Selective growth of and electricity production by marine exoelectrogenic bacteria in self-aggregated hydrogel of microbially reduced graphene oxide. C. 2:15. doi: 10.3390/c202 0015

Yoshida, N., Miyata, Y., Doi, K., Goto, Y., Nagao, Y., Tero, R., et al. (2016b). Graphene oxide-dependent growth and self-aggregation into a hydrogel complex of exoelectrogenic bacteria. Sci. Rep. 6:21867. doi: 10.1038/srep2 1867

Zhou, S., Lin, M., Zhuang, Z., Liu, P., and Chen, Z. (2019). Biosynthetic graphene enhanced extracellular electron transfer for high performance anode in microbial fuel cell. Chemosphere 232, 396-402. doi: 10.1016/j.chemosphere. 2019.05.191

Zhou, X., and Liang, F. (2014). Application of graphene/graphene oxide in biomedicine and biotechnology. Curr. Med. Chem. 21, 855-869. doi: 10.2174/ 0929867320666131119124325

Conflict of Interest: The authors declare that the research was conducted in the absence of any commercial or financial relationships that could be construed as a potential conflict of interest.

Copyright (c) 2020 Igarashi, Miyako and Kato. This is an open-access article distributed under the terms of the Creative Commons Attribution License (CC BY). The use, distribution or reproduction in other forums is permitted, provided the original author(s) and the copyright owner(s) are credited and that the original publication in this journal is cited, in accordance with accepted academic practice. No use, distribution or reproduction is permitted which does not comply with these terms. 